PROCEEDINGS OF THE

AMERICAN MATHEMATICAL SOCIETY

Volume 134, Number 3, Pages 653-663

S 0002-9939(05)08003-2

Article electronically published on July 19, 2005

\title{
THE GELFAND-KIRILLOV DIMENSION OF QUADRATIC ALGEBRAS SATISFYING THE CYCLIC CONDITION
}

\author{
FERRAN CEDÓ, ERIC JESPERS, AND JAN OKNIŃSKI
}

(Communicated by Martin Lorenz)

\begin{abstract}
We consider algebras over a field $K$ presented by generators $x_{1}, \ldots, x_{n}$ and subject to $\left(\begin{array}{c}n \\ 2\end{array}\right)$ square-free relations of the form $x_{i} x_{j}=x_{k} x_{l}$ with every monomial $x_{i} x_{j}, i \neq j$, appearing in one of the relations. It is shown that for $n>1$ the Gelfand-Kirillov dimension of such an algebra is at least two if the algebra satisfies the so-called cyclic condition. It is known that this dimension is an integer not exceeding $n$. For $n \geq 4$, we construct a family of examples of Gelfand-Kirillov dimension two. We prove that an algebra with the cyclic condition with generators $x_{1}, \ldots, x_{n}$ has Gelfand-Kirillov dimension $n$ if and only if it is of $I$-type, and this occurs if and only if the multiplicative submonoid generated by $x_{1}, \ldots, x_{n}$ is cancellative.
\end{abstract}

\section{INTRODUCTION}

In 4. Gateva-Ivanova and Van den Bergh studied the structure of monoids of left $I$-type and their algebras. These monoids originate from the work of Tate and Van den Bergh on homological properties of Sklyanin algebras [8. It was shown in [4] that a monoid of left $I$-type has a presentation with generators $x_{1}, \ldots, x_{n}$ and $\left(\begin{array}{l}n \\ 2\end{array}\right)$ relations of the form $x_{i} x_{j}=x_{k} x_{l}$ such that every monomial $x_{i} x_{j}$ with $1 \leq i, j \leq n$ appears at most once in one of the relations. Moreover, such monoids yield settheoretical solutions of the quantum Yang-Baxter equation, and the corresponding monoid algebras share many properties with commutative polynomial algebras. In particular, they are noetherian domains of finite global dimension, satisfy a polynomial identity, are Koszul, Auslander-Gorenstein, Cohen-Macaulay and have Gelfand-Kirillov dimension $n$. In 6 the monoids of left $I$-type are characterized as natural submonoids of semidirect products of the free abelian monoid of rank $n$ and the symmetric group of degree $n$. As a consequence, it is proved that a monoid is of left $I$-type if and only if it is of right $I$-type, 6, Corollary 2.3].

A monoid $S$ is said to be of skew type if it has a presentation with $n \geq 2$ generators $x_{1}, \ldots, x_{n}$ and $\left(\begin{array}{l}n \\ 2\end{array}\right)$ square-free relations of the form $x_{i} x_{j}=x_{k} x_{l}$ with every monomial $x_{i} x_{j}, i \neq j$, appearing in one of the relations (see [3], where a systematic study of these monoids and their algebras was initiated). Recall that

Received by the editors March 24, 2004 and, in revised form, October 19, 2004.

2000 Mathematics Subject Classification. Primary 16P90, 16S36, 16S15, 20M25; Secondary 16P40, 20M05, 20F05.

This work was supported in part by the Flemish-Polish bilateral agreement BIL 01/31 and KBN research grant 2P03A 03325 (Poland), the MCyT-Spain and FEDER through grant BFM200201390, and by the Generalitat de Catalunya (Grup de Recerca consolidat 2001SGR00171).

(C)2005 American Mathematical Society 
a monoid $S=\left\langle x_{1}, \ldots, x_{n}\right\rangle$ of skew type is said to be right (respectively left) nondegenerate if for every $1 \leq i, k \leq n$ there exist $1 \leq j, l \leq n$ so that $x_{i} x_{j}=x_{k} x_{l}$ $\left(x_{l} x_{k}=x_{j} x_{i}\right.$ respectively). Furthermore $S$ is said to satisfy the cyclic condition if for every relation $x_{i} x_{j}=x_{k} x_{l}$ one also has a relation $x_{i} x_{k}=x_{r} x_{l}$ for some $r$ (see [3. Lemma 2.1]). The latter is a powerful combinatorial condition that has already proved crucial in the study of monoids of $I$-type, their algebras and corresponding torsion-free groups, 4, 6. The cyclic condition is symmetric, [3, Proposition 2.1]. Hence it is easy to see that it implies left and right non-degeneracy. It was shown in [3] that for monoids $S$ satisfying the cyclic condition we have $1 \leq \mathrm{GK}(K[S]) \leq n$, where $\operatorname{GK}(K[S])$ denotes the Gelfand-Kirillov dimension of the monoid algebra $K[S]$. Furthermore there exist non-degenerate monoids $S$ of skew type on $4^{m}$ generators (for any positive $m$ ) so that $\mathrm{GK}(K[S])=1$, [1].

In this paper we prove that $\operatorname{GK}(K[S]) \geq 2$ for any monoid $S$ of skew type that satisfies the cyclic condition. For any $n \geq 4$ we construct examples of such monoids on $n$ generators with $\operatorname{GK}(K[S])=2$. Furthermore we show that $\operatorname{GK}(K[S])=n$ if and only if $S$ is of $I$-type, and this occurs if and only if $S$ is cancellative.

\section{The Gelfand-Kirillov Dimension}

Let $S=\left\langle x_{1}, x_{2}, \ldots, x_{n}\right\rangle$ be a monoid of skew type that satisfies the cyclic condition.

Let $F=\left\langle y_{1}, y_{2}, \ldots, y_{n}\right\rangle$ be the free monoid of rank $n$ and let $\pi: F \rightarrow S$ be the natural epimorphism, that is $\pi\left(y_{i}\right)=x_{i}$ for $i=1, \ldots, n$. Let $x \in S$. We say that a word $w \in F$ represents $x$ if $\pi(w)=x$.

It is known that two words $w, w^{\prime} \in F$ represent the same element $x \in S$ if and only if there exists a finite sequence of words

$$
w=w_{0}, w_{1}, w_{2}, \ldots, w_{m}=w^{\prime}
$$

such that $w_{i}$ is obtained from $w_{i-1}(i=1, \ldots, m)$ by substituting a subword $y_{j} y_{k}$ by $y_{p} y_{q}$, where $x_{j} x_{k}=x_{p} x_{q}$ is a defining relation of $S$. In this case, we say that $w_{i}$ is obtained from $w_{i-1}$ by an $S$-relation.

Lemma 2.1. If $x_{i_{1}} x_{j_{1}}=x_{j_{2}} x_{i_{2}}$ is a defining relation of $S$, then there exist positive integers $r, s$ such that $r+s \leq n$ and the submonoid $\left\langle x_{i_{1}}^{r}, x_{j_{1}}^{s}\right\rangle$ is free abelian of rank 2 .

Proof. By [3, Proposition 2.1], since $S$ satisfies the cyclic condition, there exist positive integers $r, s$ and $s+r$ different integers

$$
i_{1}, i_{2}, \ldots, i_{s}, j_{1}, j_{2}, \ldots, j_{r} \in\{1,2, \ldots, n\}
$$

such that

$$
\begin{array}{cccc}
x_{i_{1}} x_{j_{1}}=x_{j_{2}} x_{i_{2}}, & x_{i_{2}} x_{j_{1}}=x_{j_{2}} x_{i_{3}}, & \ldots, & x_{i_{s}} x_{j_{1}}=x_{j_{2}} x_{i_{1}}, \\
x_{i_{1}} x_{j_{2}}=x_{j_{3}} x_{i_{2}}, & x_{i_{2}} x_{j_{2}}=x_{j_{3}} x_{i_{3}}, & \ldots, & x_{i_{s}} x_{j_{2}}=x_{j_{3}} x_{i_{1}}, \\
\vdots & \vdots & & \vdots \\
x_{i_{1}} x_{j_{r}}=x_{j_{1}} x_{i_{2}}, & x_{i_{2}} x_{j_{r}}=x_{j_{1}} x_{i_{3}}, & \ldots, & x_{i_{s}} x_{j_{r}}=x_{j_{1}} x_{i_{1}} .
\end{array}
$$

From the relations in the first column we have

$$
x_{i_{1}}^{r} x_{j_{1}}=x_{j_{1}} x_{i_{2}}^{r}
$$

Similarly, from the other columns, we obtain

$$
x_{i_{2}}^{r} x_{j_{1}}=x_{j_{1}} x_{i_{3}}^{r}, \quad \ldots, \quad x_{i_{s}}^{r} x_{j_{1}}=x_{j_{1}} x_{i_{1}}^{r} .
$$


Hence $x_{i_{1}}^{r} x_{j_{1}}^{s}=x_{j_{1}}^{s} x_{i_{1}}^{r}$, and thus the submonoid $\left\langle x_{i_{1}}^{r}, x_{j_{1}}^{s}\right\rangle$ is abelian. Note that the only words that represent $x_{i_{1}}^{m}$ and $x_{j_{1}}^{m}$ are $y_{i_{1}}^{m}$ and $y_{j_{1}}^{m}$ respectively. Let $p, q$ be positive integers and $x=x_{i_{1}}^{r p} x_{j_{1}}^{s q}$. We claim that any word $w \in F$ that represents $x$ is of the form

$$
w=y_{i_{1}}^{n_{1}} y_{j_{k_{1}}}^{m_{1}} y_{i_{l_{2}}}^{n_{2}} y_{j_{k_{2}}}^{m_{2}} \ldots y_{i_{l_{g-1}}}^{n_{g-1}} y_{j_{k_{g}-1}}^{m_{g-1}} y_{i_{l_{g}}}^{n_{g}},
$$

where $g$ is an integer greater than $1 ; n_{1}, n_{g}$ are non-negative integers; $l_{1}=l_{g}=1$, and $n_{2}, n_{3}, \ldots, n_{g-1}, m_{1}, m_{2}, \ldots, m_{g-1}$ are positive integers such that

(i) $n_{1}+k_{1} \equiv k_{g-1}-n_{g} \equiv 1(\bmod r)$ and $l_{t+1}-l_{t} \equiv m_{t}(\bmod s)$ for all $1 \leq t \leq g-1$

(ii) if $g>2$, then $k_{u}-k_{u+1} \equiv n_{u+1}(\bmod r)$ for all $1 \leq u \leq g-2$;

(iii) $n_{1}+n_{2}+\cdots+n_{g}=r p$ and $m_{1}+m_{2}+\cdots+m_{g-1}=s q$.

Note that the word $y_{i_{1}}^{r p} y_{j_{1}}^{s q}$ represents $x$ and satisfies conditions $(i),(i i)$ and $(i i i)$. Therefore, in order to prove the claim, it is sufficient to see that given any word $w$ of the form (11) that satisfies conditions $(i),(i i)$ and $(i i i)$, all the words obtained from $w$ by an $S$-relation also satisfy conditions $(i),($ ii) and $(i i i)$. Suppose that $g=2$. In this case

$$
w=y_{i_{1}}^{n_{1}} y_{j_{k_{1}}}^{m_{1}} y_{i_{1}}^{n_{2}}
$$

with $m_{1}=s q>0, n_{1}+n_{2}=r p$ and $n_{1}+k_{1} \equiv k_{1}-n_{2} \equiv 1(\bmod r)$. If $n_{1}>0$, we can obtain by an $S$-relation (the relation $x_{i_{1}} x_{j_{k_{1}}}=x_{j_{k_{1}+1}} x_{i_{2}}$ ), the word

$$
w^{\prime}=y_{i_{1}}^{n_{1}-1} y_{j_{k_{1}+1}} y_{i_{2}} y_{j_{k_{1}}}^{m_{1}-1} y_{i_{1}}^{n_{2}}
$$

where $k_{1}+1$ is taken modulo $r$ in the set $\{1, \ldots, r\}$, and it is easy to see that $w^{\prime}$ satisfies conditions $(i),\left(\right.$ ii) and $(i i i)$. If $n_{2}>0$, we can obtain by an $S$-relation the word

$$
w^{\prime}=y_{i_{1}}^{n_{1}} y_{j_{k_{1}}}^{m_{1}-1} y_{i_{s}} y_{j_{k_{1}-1}} y_{i_{1}}^{n_{2}-1}
$$

where $k_{1}-1$ is taken modulo $r$ in the set $\{1, \ldots, r\}$, and it is easy to see that $w^{\prime}$ satisfies the conditions $(i),(i i)$ and $(i i i)$. Similarly, it is straightforward to prove that, if $g>2$, all the words $w^{\prime}$ obtained from $w$ by an $S$-relation satisfy conditions $(i),(i i)$ and $(i i i)$. Now condition $(i i i)$ implies that the submonoid $\left\langle x_{i_{1}}^{r}, x_{j_{1}}^{s}\right\rangle$ is free abelian of rank 2 .

As a direct consequence of Lemma 2.1 we get the following result.

Corollary 2.2. Let $S=\left\langle x_{1}, x_{2}, \ldots, x_{n}\right\rangle$ be a monoid of skew type that satisfies the cyclic condition. Let $m=(n-1)$ !. Then the submonoid $A=\left\langle x_{1}^{m}, \ldots, x_{n}^{m}\right\rangle$ is commutative.

Theorem 2.3. Let $S=\left\langle x_{1}, x_{2}, \ldots, x_{n}\right\rangle$ (with $n>1$ ) be a monoid of skew type that satisfies the cyclic condition. Let $A=\left\langle x_{1}^{m}, \ldots, x_{n}^{m}\right\rangle$, where $m=(n-1)$ !. If $K$ is a field, then the Gelfand-Kirillov dimension of the monoid algebra $K[S]$ is an integer such that $2 \leq \mathrm{GK}(K[S])=\mathrm{GK}(K[A]) \leq n$. Moreover, $\mathrm{GK}(K[S])$ is equal to the maximal rank $k$ of a free abelian submonoid of the form $\left\langle x_{i_{1}}^{m}, \ldots, x_{i_{k}}^{m}\right\rangle \subseteq S$.

Proof. By [3, Theorem 4.5] and the comment after [3, Proposition 2.4], $K[S]$ is a finite left and right module over the commutative subring $K[A]$, where $A=$ $\left\langle x_{1}^{p}, \ldots, x_{n}^{p}\right\rangle$ for some $p \geq 1$. The proof actually shows that we may take $p=(n-1)$ !. Hence $\mathrm{GK}(K[S])=\mathrm{GK}(K[A]) \leq n$ and it is an integer. By Lemma 2.1] we have that $2 \leq \operatorname{GK}(K[S])$. Let $P$ be a prime ideal of $K[A]$. Then the image $A_{P}$ of 
$A$ in $K[A] / P$ is a 0 -cancellative monoid. Let $C=\left\langle z_{i_{1}}^{m}, \ldots, z_{i_{r}}^{m}\right\rangle \subseteq A_{P}$ be a free abelian submonoid of maximal rank that is generated by certain images $z_{i}^{m}$ of the elements $x_{i}^{m}$. Then $B=\left\langle x_{i_{1}}^{m}, \ldots, x_{i_{r}}^{m}\right\rangle \subseteq A$ is free abelian of rank $r$. It is easy to see that the group $G_{P}$ of quotients of the cancellative semigroup of nonzero elements of $A_{P}$ is of rank $r$, whence $\operatorname{GK}(K[A] / P) \leq \mathrm{GK}\left(K\left[A_{P}\right]\right) \leq \mathrm{GK}\left(K\left[G_{P}\right]\right)=r \leq k$. Therefore $\operatorname{GK}(A) \leq k$, since the Gelfand-Kirillov and the classical Krull dimensions coincide on finitely generated commutative algebras, 7, Theorem 4.5]. The result follows.

\section{EXAmples OF Dimension two}

For $n \geq 4$, let $T^{(n)}$ be the monoid of skew type generated by $x_{1}, \ldots, x_{n}$ with defining relations

$$
\begin{aligned}
& x_{1} x_{2}=x_{3} x_{1}, \quad \ldots, \quad x_{1} x_{n-2}=x_{n-1} x_{1}, \quad x_{1} x_{n-1}=x_{2} x_{1}, \\
& x_{n} x_{1}=x_{n-1} x_{n}, \quad x_{n} x_{n-1}=x_{1} x_{n}, \\
& x_{i} x_{i+1}=x_{i+2} x_{i}, \quad \ldots, \quad x_{i} x_{n-1}=x_{n} x_{i}, \quad x_{i} x_{n}=x_{i+1} x_{i},
\end{aligned}
$$

for all $2 \leq i \leq n-2$. Note that $T^{(n)}$ satisfies the cyclic condition.

Lemma 3.1. Let $\rho$ be the least cancellative congruence on $T^{(n)}$. If $n>4$, then $x_{2} x_{1} x_{2}=x_{n} x_{1} x_{2}$ and $x_{1} \rho x_{2} \rho \ldots \rho x_{n}$.

Proof. By using the defining relations, we have

$x_{2} x_{1} x_{2}=x_{1} x_{n-1} x_{2}=x_{1} x_{2} x_{n-2}=x_{3} x_{1} x_{n-2}=x_{3} x_{n-1} x_{1}=x_{n} x_{3} x_{1}=x_{n} x_{1} x_{2}$.

Since $x_{2} x_{1} x_{2}=x_{n} x_{1} x_{2}$, it follows that $x_{2} \rho x_{n}$. Now the relations

$$
x_{2} x_{3}=x_{4} x_{2}, \quad \ldots, \quad x_{2} x_{n-1}=x_{n} x_{2}
$$

imply that $x_{2} \rho x_{3} \rho \ldots \rho x_{n}$. Since $x_{n} x_{1}=x_{n-1} x_{n}$, we also get

$$
x_{1} \rho x_{2} \rho \ldots \rho x_{n}
$$

Let $T_{n}^{\prime}$ be the subset of $T^{(n)}$ of all elements right divisible by all generators of $T^{(n)}$. Since $T^{(n)}$ is left non-degenerate, $T_{n}^{\prime}$ is an ideal of $T^{(n)}$; see [3].

Lemma 3.2. Consider $z=x_{2} x_{1} x_{2} \in T^{(n)}$. Then $z \in T_{n}^{\prime}$.

Proof. For $n=4$ we have

$$
z=x_{2} x_{1} x_{2}=x_{2} x_{3} x_{1}=x_{4} x_{2} x_{1}=x_{4} x_{1} x_{3}=x_{3} x_{4} x_{3}=x_{3} x_{1} x_{4} \in T_{n}^{\prime} .
$$

Suppose that $n>4$. By Lemma 3.1] $z=x_{n} x_{1} x_{2}$ and thus

$$
\begin{aligned}
z & =x_{n} x_{1} x_{2}=x_{n-1} x_{n} x_{2}=x_{n-1} x_{2} x_{n-1}=x_{2} x_{n-2} x_{n-1} \\
& =x_{2} x_{n} x_{n-2}=x_{3} x_{2} x_{n-2}=x_{3} x_{n-1} x_{2}=x_{n} x_{3} x_{2}=x_{n} x_{2} x_{n} \\
& =x_{2} x_{n-1} x_{n}=x_{2} x_{n} x_{1}=x_{3} x_{2} x_{1}=x_{3} x_{1} x_{n-1}=x_{1} x_{2} x_{n-1} \\
& =x_{1} x_{n} x_{2}=x_{n} x_{n-1} x_{2}=x_{n} x_{2} x_{n-2}=x_{2} x_{n-1} x_{n-2} .
\end{aligned}
$$

We claim that $z=x_{2} x_{i+1} x_{i}$ for all $n-2 \geq i \geq 3$. We prove this by induction. If $n=5$ the claim is proved. Suppose that $n>5$ and that we know $z=x_{2} x_{i+1} x_{i}$ for 
some $4 \leq i \leq n-2$. Then

$$
\begin{aligned}
z & =x_{2} x_{i+1} x_{i}=x_{2} x_{i} x_{n}=x_{i+1} x_{2} x_{n}=x_{i+1} x_{3} x_{2} \\
& =x_{3} x_{i} x_{2}=x_{3} x_{2} x_{i-1}=x_{2} x_{n} x_{i-1}=x_{2} x_{i-1} x_{n-1} \\
& =x_{i} x_{2} x_{n-1}=x_{i} x_{n} x_{2}=x_{i+1} x_{i} x_{2}=x_{i+1} x_{2} x_{i-1}=x_{2} x_{i} x_{i-1}
\end{aligned}
$$

which proves the inductive claim. It follows that $z \in T^{(n)} x_{i}$, for all $3 \leq i \leq n-2$. Since $z=x_{2} x_{1} x_{2}=x_{2} x_{3} x_{1}=x_{n-1} x_{2} x_{n-1}=x_{n} x_{2} x_{n}$, we have that $z \in T_{n}^{\prime}$.

Let $m=(n-1)$ !. By Corollary 2.2, the submonoid $A=\left\langle x_{1}^{m}, \ldots, x_{n}^{m}\right\rangle$ of $T^{(n)}$ is commutative.

Lemma 3.3. If $n>4$, then $x_{k}^{2 m} x_{j}^{2 m} x_{i}^{2 m} \in T_{n}^{\prime}$ for all $1 \leq i<j<k \leq n$.

Proof. Note that from the relations

$$
x_{1} x_{2}=x_{3} x_{1}, \quad \ldots, \quad x_{1} x_{n-2}=x_{n-1} x_{1}, \quad x_{1} x_{n-1}=x_{2} x_{1}
$$

it follows that

$$
x_{1}^{j} x_{i}=x_{j+i} x_{1}^{j}, \quad x_{1}^{n-2} x_{i}=x_{i} x_{1}^{n-2},
$$

for all $2 \leq i \leq n-1$ and $1 \leq j<n-i$. From the relations

$$
x_{n} x_{1}=x_{n-1} x_{n}, \quad x_{n} x_{n-1}=x_{1} x_{n},
$$

it follows that

$$
x_{n}^{2} x_{1}=x_{1} x_{n}^{2} \quad \text { and } \quad x_{n}^{2} x_{n-1}=x_{n-1} x_{n}^{2} .
$$

For each $2 \leq i \leq n-2$, the relations

$$
x_{i} x_{i+1}=x_{i+2} x_{i}, \quad \ldots, \quad x_{i} x_{n-1}=x_{n} x_{i}, \quad x_{i} x_{n}=x_{i+1} x_{i}
$$

imply that

$$
x_{i}^{n-i} x_{j}=x_{j} x_{i}^{n-i}
$$

for all $2 \leq i<j \leq n$.

Case 1. $1<i<j<k \leq n$. In this case it is easy to see that

$$
x_{k} x_{j}^{k-j-1}=x_{j}^{k-j-1} x_{j+1}
$$

and

$$
x_{j} x_{i}^{j-i+1}=x_{i}^{j-i+1} x_{n-1} .
$$


Then we have

$$
\begin{aligned}
& \left.x_{k}^{2 m} x_{j}^{2 m} x_{i}^{2 m}=x_{j}^{k-j-1} x_{j+1}^{2 m} x_{j}^{2 m-k+j+1} x_{i}^{2 m} \quad \text { (by (6) }\right) \\
& =x_{j}^{k-j-1} x_{j+1}^{2 m} x_{j}^{m} x_{i}^{2 m} x_{j}^{m-k+j+1} \quad \text { (by (15) } \\
& =x_{j}^{k-j-1} x_{j}^{m} x_{j+1}^{2 m} x_{i}^{2 m} x_{j}^{m-k+j+1} \quad \text { (by (5-) } \\
& =x_{j}^{k-j-1} x_{i}^{j-i+1} x_{n-1}^{m} x_{n}^{2 m} x_{i}^{2 m-j+i-1} x_{j}^{m-k+j+1} \quad \text { (by (7)) } \\
& \left.=x_{j}^{k-j-1} x_{i}^{j-i+1} x_{n} x_{1}^{m} x_{n}^{2 m-1} x_{i}^{2 m-j+i-1} x_{j}^{m-k+j+1} \quad \text { (by (3) }\right) \\
& =x_{j}^{k-j-1} x_{i}^{j-i+1} x_{n} x_{1}^{m} x_{n}^{2} x_{i}^{m} x_{n}^{2 m-3} x_{i}^{m-j+i-1} x_{j}^{m-k+j+1} \quad \text { (by (15) } \\
& =x_{j}^{k-j-1} x_{i}^{j-i+1} x_{n}^{3} x_{1}^{m} x_{i}^{m} x_{n}^{2 m-3} x_{i}^{m-j+i-1} x_{j}^{m-k+j+1} \quad \text { (by (4)) } \\
& =x_{j}^{k-j-1} x_{i}^{j-i+1} x_{n}^{3} x_{1}^{m-n+i} x_{2}^{m} x_{1}^{n-i} x_{n}^{2 m-3} \\
& \cdot x_{i}^{m-j+i-1} x_{j}^{m-k+j+1} \quad\left(\text { since } x_{2} x_{1}^{n-i}=x_{1}^{n-i} x_{i}\right) \\
& =x_{j}^{k-j-1} x_{i}^{j-i+1} x_{n} x_{1}^{m-n+i-1} x_{n}^{2} x_{1} x_{2}^{m} x_{1}^{n-i} x_{n}^{2 m-3} \\
& \cdot x_{i}^{m-j+i-1} x_{j}^{m-k+j+1} \quad \text { (by (44) } \\
& =\left(x_{j}^{k-j-1} x_{i}^{j-i+1} x_{n} x_{1}^{m-n+i-1} x_{n}\right) z\left(x_{2}^{m-1} x_{1}^{n-i} x_{n}^{2 m-3}\right. \\
& \left.\cdot x_{i}^{m-j+i-1} x_{j}^{m-k+j+1}\right) .
\end{aligned}
$$

By Lemma 3.2 we know that $z \in T_{n}^{\prime}$. Since $T_{n}^{\prime}$ is an ideal of $T^{(n)}$, it follows that $x_{k}^{2 m} x_{j}^{2 m} x_{i}^{2 m} \in T_{n}^{\prime}$.

Case 2. $1=i<j<k \leq n$ and $j<n-1$. Then we have

$$
\begin{aligned}
& x_{k}^{2 m} x_{j}^{2 m} x_{1}^{2 m}=x_{j}^{k-j-1} x_{j+1}^{2 m} x_{j}^{2 m-k+j+1} x_{1}^{2 m} \text { (by (6) ) } \\
& \left.=x_{j}^{k-j-1} x_{j+1}^{2 m} x_{j}^{m} x_{1}^{2 m} x_{j}^{m-k+j+1} \quad \text { (by (2) }\right) \\
& =x_{j}^{k-j-1} x_{j}^{m} x_{j+1}^{2 m} x_{1}^{2 m} x_{j}^{m-k+j+1} \quad \text { (by (5) ) } \\
& =x_{j}^{k-j-1} x_{j}^{m} x_{j+1} x_{1}^{2 m} x_{j+1}^{2 m-1} x_{j}^{m-k+j+1} \quad \text { (by (2)) } \\
& =x_{j}^{k-j-1} x_{j}^{m} x_{1}^{j-2} x_{3} x_{1}^{2 m-j+2} x_{j+1}^{2 m-1} x_{j}^{m-k+j+1} \quad \text { (by (2) ) } \\
& =x_{j}^{k-j-1} x_{1}^{j-2} x_{2}^{m} x_{3} x_{1}^{2 m-j+2} x_{j+1}^{2 m-1} x_{j}^{m-k+j+1} \quad \text { (by (2)) } \\
& =\left(x_{j}^{k-j-1} x_{1}^{j-2} x_{2}^{m-1}\right) z\left(x_{1}^{2 m-j+1} x_{j+1}^{2 m-1} x_{j}^{m-k+j+1}\right) .
\end{aligned}
$$

By Lemma 3.2, $x_{k}^{2 m} x_{j}^{2 m} x_{i}^{2 m} \in T_{n}^{\prime}$ in this case.

Case 3. $i=1, j=n-1$ and $k=n$. Then we have

$$
\begin{aligned}
x_{n}^{2 m} x_{n-1}^{2 m} x_{1}^{2 m} & =x_{n}^{2 m} x_{n-1} x_{1}^{2 m} x_{n-1}^{2 m-1} \quad \text { (by (2)) } \\
& =x_{n}^{2 m} x_{1}^{n-3} x_{2} x_{1}^{2 m-n+3} x_{n-1}^{2 m-1} \quad \text { (by (2) ) } \\
& =x_{n}^{2 m-1} x_{n-1}^{n-4} x_{n} x_{1} x_{2} x_{1}^{2 m-n+3} x_{n-1}^{2 m-1} \quad \text { (by (3) ) } \\
& =\left(x_{n}^{2 m-1} x_{n-1}^{n-4}\right) z\left(x_{1}^{2 m-n+3} x_{n-1}^{2 m-1}\right) .
\end{aligned}
$$

Again, by Lemma 3.2, $x_{k}^{2 m} x_{j}^{2 m} x_{i}^{2 m} \in T_{n}^{\prime}$ in this case.

Therefore $x_{k}^{2 m} x_{j}^{2 m} x_{i}^{2 m} \in T_{n}^{\prime}$ for all $1 \leq i<j<k \leq n$.

Theorem 3.4. Let $K$ be a field. Then $\operatorname{GK}\left(K\left[T^{(n)}\right]\right)=2$ for all $n \geq 4$. 
Proof. For $n=4$ the result follows from [5, Proposition 2.1], because $T^{(4)}$ coincides with the monoid $C^{(1)}$ of $[5$. Suppose that $n>4$. As above, let $m=(n-1)$ !. From [3, Proposition 6.3] we know that $\left(T_{n}^{\prime}\right)^{q} I(\rho)=0$ for some $q$, where $I(\rho)$ is the ideal of $K\left[T^{(n)}\right]$ determined by the least cancellative congruence $\rho$ on $T^{(n)}$. In particular, by Lemma 3.1, $x_{k}^{m}-x_{j}^{m} \in I(\rho)$ for all $k, j$. Therefore, from Lemma 3.3 it follows that

$$
x_{k}^{2 m q} x_{j}^{2 m q} x_{i}^{2 m q}\left(x_{k}^{m}-x_{j}^{m}\right)=0,
$$

for all $1 \leq i<j<k \leq n$, which implies that $x_{k}^{m}, x_{j}^{m}, x_{i}^{m}$ do not generate a free abelian semigroup. Therefore Theorem 2.3 implies that $\operatorname{GK}\left(K\left[T^{(n)}\right]\right)=2$.

Corollary 3.5. For any integers $n \geq 4$ and $2 \leq j \leq n$, there exists a monoid $M=\left\langle x_{1}, x_{2}, \ldots, x_{n}\right\rangle$ of skew type, satisfying the cyclic condition and such that $\operatorname{GK}(K[M])=j$ for any field $K$.

Proof. If $j=n$, then the free abelian monoid of rank $n, M=\mathrm{FaM}_{n}$, satisfies the conditions.

Suppose that $j=n-1$. By [5], there exists a monoid $A$ of skew type with 4 generators that satisfies the cyclic condition such that, for any field $K, \operatorname{GK}(K[A])=$ 3. Let $M=A \times \mathrm{FaM}_{n-4}$. Then it is easy to see that $M$ is a monoid of skew type with $n$ generators that satisfies the cyclic condition. Since $K[M]$ is the polynomial algebra over $K[A]$ with $n-4$ indeterminates, by [7, Example 3.6], $\operatorname{GK}(K[M])=$ $3+(n-4)=n-1$.

Suppose that $j \leq n-2$. Let $M=T^{(n-j+2)} \times \mathrm{FaM}_{j-2}$. It is easy to see that $M$ is a monoid of skew type with $n$ generators that satisfies the cyclic condition. Since $K[M]$ is the polynomial algebra over $K\left[T^{(n-j+2)}\right]$ with $j-2$ indeterminates, by [7, Example 3.6], $\operatorname{GK}(K[M])=\operatorname{GK}\left(K\left[T^{(n-j+2)}\right]\right)+(j-2)$. By Theorem 3.4, $\operatorname{GK}(K[M])=j$.

\section{I-TYPE MONOIDS}

Let $\mathrm{FaM}_{n}$ be the multiplicative free abelian monoid of rank $n$ with basis $u_{1}, \ldots$, $u_{n}$. Recall that a monoid $S$ generated by $x_{1}, \ldots, x_{n}$ is said to be of left $I$-type if there exists a bijection (called a left $I$-structure)

$$
v: \mathrm{FaM}_{n} \rightarrow S
$$

such that

$$
v(1)=1 \quad \text { and } \quad\left\{v\left(u_{1} a\right), \ldots, v\left(u_{n} a\right)\right\}=\left\{x_{1} v(a), \ldots, x_{n} v(a)\right\}
$$

for all $a \in \mathrm{FaM}_{n}$. As mentioned in the introduction, it is proved in [6] that a monoid $S$ is of left $I$-type if and only if it is of right $I$-type. So we call a monoid of left or right $I$-type simply a monoid of $I$-type.

Let $S=\left\langle x_{1}, x_{2}, \ldots, x_{n}\right\rangle$ be a monoid of skew type. Let $X=\left\{x_{1}, x_{2}, \ldots, x_{n}\right\}$. As in [6], we define the associated bijective map $r: X \times X \rightarrow X \times X$ by

$$
r\left(x_{i}, x_{j}\right)=\left(x_{k}, x_{l}\right)
$$

if $x_{i} x_{j}=x_{k} x_{l}$ is a defining relation of $S$, and $r\left(x_{i}, x_{i}\right)=\left(x_{i}, x_{i}\right)$. For each $x \in X$, we also denote by $f_{x}: X \rightarrow X$ and $g_{x}: X \rightarrow X$ the mappings defined by $f_{x}\left(x_{i}\right)=$ $p_{1}\left(r\left(x, x_{i}\right)\right)$ and $g_{x}\left(x_{i}\right)=p_{2}\left(r\left(x_{i}, x\right)\right)$, where $p_{1}$ and $p_{2}$ denote the projections onto the first and second component respectively. So $r\left(x_{i}, x_{j}\right)=\left(f_{x_{i}}\left(x_{j}\right), g_{x_{j}}\left(x_{i}\right)\right)$. Suppose that $S$ is right non-degenerate. So $f_{x}$ is bijective for all $x \in X$. We denote by $\sigma_{i} \in \operatorname{Sym}_{n}$ the permutation defined by $f_{x_{i}}\left(x_{j}\right)=x_{\sigma_{i}(j)}$. 
The next result is a partial generalization of Proposition 2.2(c) of [2].

Theorem 4.1. Let $S=\left\langle x_{1}, x_{2}, \ldots, x_{n}\right\rangle$ be a right non-degenerate monoid of skew type. Then the following conditions are equivalent.

(i) $S$ is of I-type.

(ii) $\sigma_{i} \circ \sigma_{\sigma_{i}^{-1}(j)}=\sigma_{j} \circ \sigma_{\sigma_{j}^{-1}(i)}$ for all $i, j$.

(iii) For every defining relation $x_{i} x_{j}=x_{k} x_{l}$ of $S$ we have $\sigma_{i} \circ \sigma_{j}=\sigma_{k} \circ \sigma_{l}$.

Proof. We denote by $r_{i}: X^{3} \rightarrow X^{3}$, for $i=1,2$, the mappings defined by $r_{1}=$ $r \times i d_{X}$ and $r_{2}=i d_{X} \times r$. Then

$$
\begin{aligned}
& \left(r_{1} \circ r_{2} \circ r_{1}\right)\left(x_{i}, x_{j}, x_{k}\right)=\left(r_{1} \circ r_{2}\right)\left(x_{\sigma_{i}(j)}, x_{\sigma_{\sigma_{i}(j)}^{-1}(i)}, x_{k}\right) \\
& =r_{1}\left(x_{\sigma_{i}(j)}, x_{\sigma_{\sigma_{i}(j)}^{-1}(i)}(k), x_{\sigma_{\sigma_{\sigma_{i}(j)}^{-1}}^{-1}(k)}\left(\sigma_{\sigma_{i}(j)}^{-1}(i)\right)\right)
\end{aligned}
$$

and

$$
\begin{aligned}
\left(r_{2} \circ r_{1} \circ r_{2}\right)\left(x_{i}, x_{j}, x_{k}\right) & =\left(r_{2} \circ r_{1}\right)\left(x_{i}, x_{\sigma_{j}(k)}, x_{\sigma_{\sigma_{j}(k)}^{-1}(j)}\right) \\
& =r_{2}\left(x_{\sigma_{i}\left(\sigma_{j}(k)\right)}, x_{\sigma_{\sigma_{i}\left(\sigma_{j}(k)\right)}^{-1}(i)}, x_{\sigma_{\sigma_{j}(k)}^{-1}(j)}\right) .
\end{aligned}
$$

Recall from [6. Corollary 3.1] that $S$ is of $I$-type if and only if $r$ yields a solution of the quantum Yang-Baxter equation, that is $r_{1} \circ r_{2} \circ r_{1}=r_{2} \circ r_{1} \circ r_{2}$. Therefore, if $S$ is of $I$-type, then by (8) and (9), we have

$$
\sigma_{\sigma_{i}(j)}\left(\sigma_{\sigma_{\sigma_{i}(j)}^{-1}(i)}(k)\right)=\sigma_{i}\left(\sigma_{j}(k)\right),
$$

for all $i, j, k$. Thus

$$
\sigma_{\sigma_{i}(j)} \circ \sigma_{\sigma_{\sigma_{i}(j)}^{-1}(i)}=\sigma_{i} \circ \sigma_{j}
$$

for all $i, j$. By putting $j^{\prime}=\sigma_{i}(j)$, we can write (10) as

$$
\sigma_{j^{\prime}} \circ \sigma_{\sigma_{j^{\prime}}^{-1}(i)}=\sigma_{i} \circ \sigma_{\sigma_{i}^{-1}\left(j^{\prime}\right)},
$$

for all $i, j^{\prime}$. Therefore $(i i)$ is a consequence of $(i)$.

Suppose that

$$
\sigma_{i} \circ \sigma_{\sigma_{i}^{-1}(j)}=\sigma_{j} \circ \sigma_{\sigma_{j}^{-1}(i)},
$$

for all $i, j$. We will prove that $r$ yields a solution of the quantum Yang-Baxter equation and thus $S$ is of $I$-type. By (8) and (9), it is sufficient to prove the following equalities:

(a) $\sigma_{\sigma_{i}(j)}\left(\sigma_{\sigma_{\sigma_{i}(j)}^{-1}(i)}(k)\right)=\sigma_{i}\left(\sigma_{j}(k)\right)$;

(b) $\sigma_{\sigma_{\sigma_{i}(j)}\left(\sigma_{\sigma_{\sigma_{i}(j)}^{-1}(i)}^{-1}(k)\right)}\left(\sigma_{i}(j)\right)=\sigma_{\sigma_{\sigma_{i}\left(\sigma_{j}(k)\right)}^{-1}(i)}\left(\sigma_{\sigma_{j}(k)}^{-1}(j)\right)$;

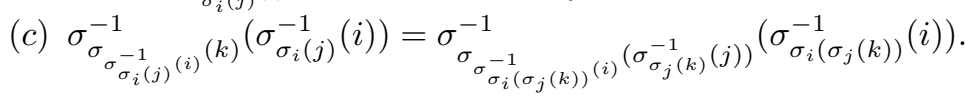

The equality $(a)$ follows from

$$
\sigma_{j^{\prime}} \circ \sigma_{\sigma_{j^{\prime}}^{-1}(i)}=\sigma_{i} \circ \sigma_{\sigma_{i}^{-1}\left(j^{\prime}\right)},
$$

with $j^{\prime}=\sigma_{i}(j)$. By $(a)$, the equality $(b)$ is equivalent to

$$
\sigma_{\sigma_{i}\left(\sigma_{j}(k)\right)}^{-1}\left(\sigma_{i}(j)\right)=\sigma_{\sigma_{\sigma_{i}\left(\sigma_{j}(k)\right)}^{-1}(i)}\left(\sigma_{\sigma_{j}(k)}^{-1}(j)\right),
$$


and the latter follows from our assumption

$$
\sigma_{l}^{-1} \circ \sigma_{i}=\sigma_{\sigma_{l}^{-1}(i)} \circ \sigma_{\sigma_{i}^{-1}(l)}^{-1},
$$

with $l=\sigma_{i}\left(\sigma_{j}(k)\right)$. By $(a)$, the equality $(c)$ is equivalent to

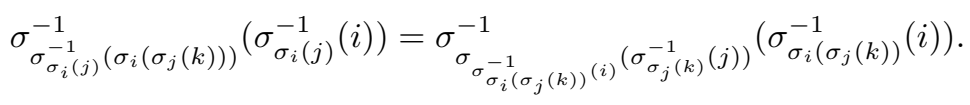

In view of (11), this equality is equivalent to

$$
\sigma_{\sigma_{\sigma_{i}(j)}^{-1}\left(\sigma_{i}\left(\sigma_{j}(k)\right)\right)}^{-1}\left(\sigma_{\sigma_{i}(j)}^{-1}(i)\right)=\sigma_{\sigma_{\sigma_{i}\left(\sigma_{j}(k)\right)}^{-1}\left(\sigma_{i}(j)\right)}^{-1}\left(\sigma_{\sigma_{i}\left(\sigma_{j}(k)\right)}^{-1}(i)\right),
$$

and the latter follows from our assumption

$$
\sigma_{\sigma_{j^{\prime}}^{-1}(l)}^{-1} \circ \sigma_{j^{\prime}}^{-1}=\sigma_{\sigma_{l}^{-1}\left(j^{\prime}\right)}^{-1} \circ \sigma_{l}^{-1}
$$

with $l=\sigma_{i}\left(\sigma_{j}(k)\right)$ and $j^{\prime}=\sigma_{i}(j)$. Hence $r$ yields a solution of the quantum Yang-Baxter equation and (ii) implies $(i)$.

Finally, notice that $x_{i} x_{p}=x_{j} x_{q}$ if and only if $\sigma_{i}(p)=j$ and $\sigma_{j}(q)=i$. The latter is equivalent to $\sigma_{i}^{-1}(j)=p$ and $\sigma_{j}^{-1}(i)=q$. Hence, saying that $\sigma_{i} \sigma_{p}=$ $\sigma_{j} \sigma_{q}$ whenever $x_{i} x_{p}=x_{j} x_{q}$ is equivalent to saying that $\sigma_{i} \sigma_{\sigma_{i}^{-1}(j)}=\sigma_{j} \sigma_{\sigma_{j}^{-1}(i)}$. So conditions (ii) and (iii) are equivalent. This completes the proof.

\section{The Dimension $n$ CASE}

Let $S=\left\langle x_{1}, x_{2}, \ldots, x_{n}\right\rangle$ be a monoid of skew type that satisfies the cyclic condition. In this section we study the second extreme case, namely the case where $\operatorname{GK}(K[S])=n$ for any field $K$. As in Section 4 we define $\sigma_{i} \in \operatorname{Sym}_{n}$ by

$$
\sigma_{i}(j)=\left\{\begin{array}{lll}
i & \text { if } \quad j=i, \\
k & \text { if } \quad x_{i} x_{j}=x_{k} x_{l} \quad \text { is a defining relation of } S .
\end{array}\right.
$$

Let $m=(n-1)$ !. Since $S$ satisfies the cyclic condition, for all $i, j$ we have that

$$
x_{i} x_{j}^{m}=x_{\sigma_{i}(j)}^{m} x_{i} .
$$

Theorem 5.1. Let $S=\left\langle x_{1}, x_{2}, \ldots, x_{n}\right\rangle$ be a monoid of skew type that satisfies the cyclic condition. Let $K$ be a field. Then the following conditions are equivalent:

(i) $\operatorname{GK}(K[S])=n$.

(ii) $S$ is of I-type.

(iii) $S$ is cancellative.

Proof. $(i) \Rightarrow(i i)$. Suppose that $\operatorname{GK}(K[S])=n$. Let $m=(n-1)$ !. We know that $A=\left\langle x_{1}^{m}, \ldots, x_{n}^{m}\right\rangle$ is abelian. Moreover $\operatorname{GK}(K[A])=\operatorname{GK}(K[S])=n$ by Theorem 2.3. This implies that $A$ is a free abelian monoid of rank $n$. Indeed, otherwise the natural map $K\left[y_{1}, \ldots, y_{n}\right] \rightarrow K[A]$ has a nontrivial kernel, whence the classical Krull dimension of $K[A]$ is smaller than $n$, while it is equal to the Gelfand-Kirillov dimension; see [7, Theorem 4.5].

Suppose that $x_{i} x_{j}=x_{k} x_{l}$ is a defining relation of $S$. Then for all $t \in\{1, \ldots, n\}$ we have, by (12),

$$
x_{i} x_{j} x_{t}^{m}=x_{i} x_{\sigma_{j}(t)}^{m} x_{j}=x_{\sigma_{i}\left(\sigma_{j}(t)\right)}^{m} x_{i} x_{j} .
$$


Also we have

$$
x_{k} x_{l} x_{t}^{m}=x_{k} x_{\sigma_{l}(t)}^{m} x_{l}=x_{\sigma_{k}\left(\sigma_{l}(t)\right)}^{m} x_{k} x_{l} .
$$

Since

$$
x_{i} x_{j} x_{j}^{m-1} x_{i}^{m-1}=x_{i} x_{j}^{m} x_{i}^{m-1}=x_{k}^{m} x_{i} x_{i}^{m-1}=x_{k}^{m} x_{i}^{m},
$$

multiplying the two previous equalities by $x_{j}^{m-1} x_{i}^{m-1}$ on the right, we get

$$
x_{\sigma_{i}\left(\sigma_{j}(t)\right)}^{m} x_{k}^{m} x_{i}^{m}=x_{\sigma_{k}\left(\sigma_{l}(t)\right)}^{m} x_{k}^{m} x_{i}^{m} .
$$

Since $A$ is free abelian, this implies that

$$
\sigma_{i}\left(\sigma_{j}(t)\right)=\sigma_{k}\left(\sigma_{l}(t)\right)
$$

By Theorem 4.1, $S$ is of $I$-type.

$(i i) \Rightarrow(i)$. The definition of a monoid of $I$-type implies that the growth function of $S$ is the same as that of a free abelian monoid of rank $n$. Hence $\operatorname{GK}(K[S])=n$.

$($ ii $) \Rightarrow($ iii). This follows from [4, Corollary 1.5].

$($ iii $) \Rightarrow(i i)$. Suppose that $x_{i} x_{j}=x_{k} x_{l}$ is a defining relation of $S$. Then for all $t \in\{1, \ldots, n\}$, as in the proof of the implication $(i) \Rightarrow(i i)$ we get

$$
x_{\sigma_{i}\left(\sigma_{j}(t)\right)}^{m} x_{i} x_{j}=x_{\sigma_{k}\left(\sigma_{l}(t)\right)}^{m} x_{k} x_{l} .
$$

Since $S$ is cancellative, this implies that

$$
x_{\sigma_{i}\left(\sigma_{j}(t)\right)}^{m}=x_{\sigma_{k}\left(\sigma_{l}(t)\right)}^{m} .
$$

By the form of the defining relations of $S$, it is then clear that

$$
\sigma_{i}\left(\sigma_{j}(t)\right)=\sigma_{k}\left(\sigma_{l}(t)\right)
$$

By Theorem 4.1 $S$ is of $I$-type.

Corollary 5.2. Let $S$ be a monoid of skew type. Then $S$ is of I-type if and only if $S$ is cancellative and satisfies the cyclic condition.

Proof. Suppose that $S=\left\langle x_{1}, \ldots, x_{n}\right\rangle$ is of $I$-type. By [4, Theorem 1.3], the associated map $r: X^{2} \rightarrow X^{2}$, where $X=\left\{x_{1}, \ldots, x_{n}\right\}$, yields a solution of the quantum Yang-Baxter equation. By [6, Corollary 3.1], $S$ satisfies the cyclic condition. Now the result follows from Theorem 5.1 .

\section{REFERENCES}

[1] F. Cedó, E. Jespers and J. Okniński, Semiprime quadratic algebras of Gelfand-Kirillov dimension one, J. Algebra Appl. 3(2004), 283-300. MR2096451

[2] P. Etingof, T. Schedler and A. Soloviev, Set-theoretical solutions to the quantum Yang-Baxter equation, Duke Math. J. 100(1999), 169-209. MR1722951 (2001c:16076)

[3] T. Gateva-Ivanova, E. Jespers and J. Okniński, Quadratic algebras of skew type and the underlying semigroups, J. Algebra 270(2003), 635-659. MR2019633 (2004m:16039)

[4] T. Gateva-Ivanova and M. Van den Bergh, Semigroups of I-Type, J. Algebra 206(1998), 97-112. MR 1637256 (99h:20090)

[5] E. Jespers and J. Okniński, Quadratic algebras of skew type satisfying the cyclic condition, Int. J. Algebra and Computation 14(2004), 479-498. MR2084381

[6] E. Jespers and J. Okniński, Monoids and groups of I-type, Algebras and Representation Theory, to appear. 
[7] G.R. Krause and T.H. Lenagan, Growth of Algebras and Gelfand-Kirillov Dimension, Revised edition. Graduate Studies in Mathematics, 22. American Mathematical Society, Providence, RI, 2000. MR1721834 (2000j:16035)

[8] J. Tate and M. Van den Bergh, Homological properties of Sklyanin algebras, Invent. Math. 124(1996), 619-647. MR1369430 (98c:16057)

Departament de Matemàtiques, Universitat Autònoma de Barcelona, 08193 BellateRRA (BARCELONa), Spain

E-mail address: cedo@mat.uab.es

Department of Mathematics, Vrije Universiteit Brussel, Pleinlaan 2, 1050 Brussel, BELGiUM

E-mail address: ef jesper@vub.ac.be

Institute of Mathematics, Warsaw University, Banacha 2, 02-097 Warsaw, Poland

E-mail address: okninski@mimuw.edu.pl 